\title{
Establishment of a fluorescence resonance energy transfer-based bioassay for detecting dioxin-like compounds
}

\author{
Chi-Iou Lin · Chun-Hung Hsieh · Stanley Shiao-Ying Lee · Wei-Shan Lee • \\ Gou-Ping Chang-Chien · Chien-Yuan Pan · Hsinyu Lee
}

Received: 16 January 2008/ Accepted: 22 June 2008/Published online: 5 July 2008

(C) National Science Council Taipei 2008

\begin{abstract}
Dioxins comprise a group of compounds which contain a double aromatic ring-like structure. They are among the most prevalent and toxic environmental pollutants. Accumulation of dioxins in human tissues poses a potential threat to human health. Currently, analytical chemical procedures dominate dioxin-detection protocols. In this study, we established a fluorescence resonance energy transfer (FRET)-based dioxin-detection bioassay. Aryl hydrocarbon receptor (AHR) and AHR nuclear translocator (ARNT) fused-cyan fluorescent protein (CFP) and -yellow fluorescent protein (YFP) constructed were transiently co-transfected into rat hepatoma cell line, H4IIEC3 cells. Our results showed that no FRET signals were detected in AHR-CFP- and ARNT-YFP-transfected H4IIEC3 cells. However, dioxin treatments upregulated FRET signals in these transfected cells in a dose-dependent manner. This work highlighted the potential of FRET technique in the detection of dioxin-like compounds.
\end{abstract}

C.-I. Lin · C.-H. Hsieh · C.-Y. Pan · H. Lee

Institute of Zoology, National Taiwan University, No. 1,

Roosevelt Rd., Sec. 4, Taipei 106, Taiwan, ROC

S. S.-Y. Lee

Chi-Hsien Clinic, No. 356, Chi-Hsien Rd., Taipei County 247,

Taiwan, ROC

W.-S. Lee - G.-P. Chang-Chien

Department of Chemical and Materials Engineering, Cheng-Shiu University, No. 840, Chengcing Rd, Niaosong Township,

Kaohsiung 833, Taiwan, ROC

H. Lee $(\square)$

Department of Life Science, National Taiwan University,

Roosevelt Rd., Sec. 4, Taipei 106, Taiwan, ROC

e-mail: hsinyu@ntu.edu.tw
Keywords Dioxin - AHR - ARNT .

Fluorescence resonance energy transfer

\section{Introduction}

2,3,7,8-Tetrachlorodibenzo-p-dioxin (TCDD) is a potent teratogen contaminant that belongs to a class of chemicals known as polyhalogenated aromatic hydrocarbons (PAHs) [1]. Dioxin contains approximately 210 different derivatives including 75 kinds of polychlorinated dibenzo-p-dioxins (PCDDs) and 135 kinds of polychlorinated dibenzofurans (PCDFs) [2]. Among these numerous dioxin derivatives, 2,3,7,8-tetrachlorodibenzo-p-dioxin (TCDD) is the most toxic [3]. TCDD was first observed from persistent side products [4]. Moreover, a previous study discovered that dioxin can be produced as a stable component after burning garbage containing chloride compounds [5]. Dioxin is widely distributed in soils and easily transported by air. With its high hydrophobic characteristics, up to $99 \%$ of the human body absorbs dioxin from the major route of food intake. Since dioxin has a structure and physiological properties similar to hormones [6], excessive intake of dioxin can seriously disrupt metabolism mechanisms, interfere with hormone systems, lead to immune and neuronal system destruction, induce cancer, and cause sterility and fetal death [7-9]. The International Agency for Research on Cancer (IARC) has declared 2,3,7,8-TCDD a specific carcinogen [10].

To the present, utilization of high-resolution gas chromatography with high-resolution mass spectrometry (HRGC/ MS) is recognized as the most efficient way for determining dioxin compounds including TCDD [11]. However, this detection system is very expensive, and the procedures are not easy to carry out. In addition, large-scale sample preparation and subsequent instrumental analysis require large amounts of 
human resources and usually take several days. Although HRGC/MS provides precise measurements and can detect each suspected TCDD-contaminating sample, various drawbacks of this technique limit its availability for large-scale sample screening. Therefore, easily manipulated, cost-effective, and time-saving analytical methods are necessary for dealing with large numbers of samples.

Bioassays are a new concept for detecting environmental pollutants including dioxin. TCDD and its structurally related compounds affect multiple biological functions through transducing the aryl hydrocarbon receptor (AHR)-mediated signaling pathway $[12,13]$. AHR is a resident protein found in the cytoplasm which forms a complex with two molecules of HSP90, a chaperone protein. In response to dioxin, the AHR-HSP90 complex is disassociated [14], and AHR is subsequently translocated into the nucleus for binding to the AHR nuclear translocator (ARNT) [15-17]. The AHRARNT complex is a transcription activator which interacts with a specific dioxin-responsive element (DRE), CYP1A1 gene expression, in response to dioxin [18]. During the past two decades, a variety of bioassays for detecting dioxins based on TCDD-mediated signal transduction mechanisms have been established. A previous study indicated the use of an enzyme-linked immunosorbent assay (ELISA) [19]. By generating monoclonal antibodies specific for 2,3,7,8TCDD, this ELISA-based detection system can detect concentrations up to the nanomolar level [20]. A chemically activated luciferase gene expression (CALUX) bioassay has also been demonstrated as an available dioxin detection system [21]. In our previous study, we established a CALUX assay-based dioxin bioassay, which was developed by cloning the mouse CYP1A1 gene in front of the luciferase reporter gene [22]. By obtaining a stable cell line expressing luciferase incorporated into CYP1A1, we easily detected dioxin concentrations by evaluating the luminescence intensity. Recently, a study reported that the (EC50) of the CYP1A1-CALUX bioassay could reach $15.6 \mathrm{pM}$, and so this can efficiently serve as an alternative bioassay method for high-throughput analysis of large numbers of suspected dioxin-contaminated samples [23]. The aryl hydrocarbon hydroxylase (AHH)/ethoxyresorufin- $O$-deethylase (EROD) bioassay is another commonly used method for detecting dioxin [24]. By measuring AHR-mediated cytochrome P450 1A induction in the H4IIE rat hepatoma cell line, the EC50 of the AHH/EROD bioassay could reach $5.6 \mathrm{pM}$ [25]. Although the CYP1A1-CALUX and AHH/EROD bioassays can detect dioxin down to the picomolar level, which represents a dramatic improvement over ELISA bioassays, these methods usually take $4-24 \mathrm{~h}$ to carry out the entire process.

The florescence resonance energy transfer (FRET) technique can precisely evaluate interactions between two molecules. To evaluate the fluorescence energy transfer between mCerulean and mCitrine, the use of cyan-fluorescent protein (CFP) as an energy donor and yellow-fluorescent protein (YFP) as an energy acceptor has been reported as the most suitable combination for FRET signal detection [26]. A high-energy CFP excited by 458-nm light will emit a 514-nm signal which excites an adjacent YFP. Therefore, the FRET signal can be detected. Evaluation of the FRET signal intensity is suitable for detecting the two-molecule association [27]. A previous study indicated that TCDD-induced AHRCFP and Hsp90-YFP disassociation decreased the FRET signal in breast cancer cells [28], which implied that the FRET technique might be available for dioxin detection.

In the present study, we constructed an AHR-CFP- and ARNT-YFP-expressing H4IIEC3 rat hepatoma cell line. By detecting the CFP, YFP, or FRET signal intensity, we observed dioxin-induced FRET signals in H4IIEC3 cells in a dose-dependent manner. These results indicated that using FRET as a dioxin-detection bioassay was successful, and this is a suitable method to initially screen for dioxinlike compounds.

\section{Materials and methods}

\section{Reagents}

Cell culture dishes were purchased from Corning Life Sciences (Corning, NY). Lipofectamine 2000 and cell culture reagents were from Invitrogen (Carlsbad, CA). Restriction enzymes and T4 DNA ligase used in the DNA manipulation were purchased from New England Biolabs (Beverly, MA). The pECFP-C1 and pEYFP-C1 vectors were from BD Biosciences Clontech (Palo Alto, CA).

\section{Plasmid construction}

Construction of the hAHR-CFP plasmid: The human AHR gene was amplified by PCR using a Bgl II forward primer (5' AGATCTATGAACAGCAGCAGCGCCAA-3') and a Sal I reverse primer (5'-GTCGACTTACAGGAATCCACTGGATG-3'). The 2.5-kb fragment encoding the full-length AHR was digested with Bgl II and Sal I. The resulting fragment was ligated to the expression vector, pECFP-C1 (Fig. 1). Construction of the hARNT-YFP plasmid: The human ARNT gene was amplified by PCR using a Bgl II forward primer (5'-AGATCTATGGCGGCGACTACTGC CAA-3 ${ }^{\prime}$ ) and a Sal I reverse primer (5'-GTCGACCTATTCTGAAAAGGGGGGAA- $3^{\prime}$ ). The $2.3-\mathrm{kb}$ PCR product encoding the full-length ARNT was digested with $\mathrm{Bgl}$ II and Sal I. The resulting fragment was ligated to the expression vector, $\mathrm{pEYFP-C1}$ (Fig. 1). Both constructs were confirmed by DNA sequencing (Mission Biotech, Taipei, Taiwan). 
A Schematic of the FRET-based bioassay

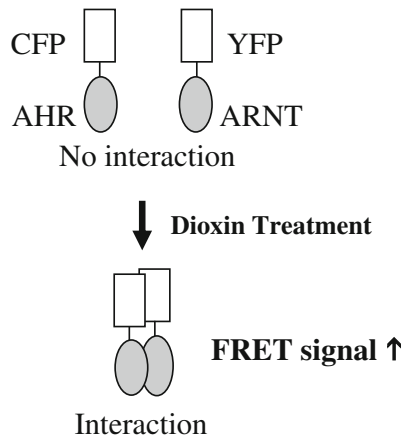

B Summary of chimeric constructs used

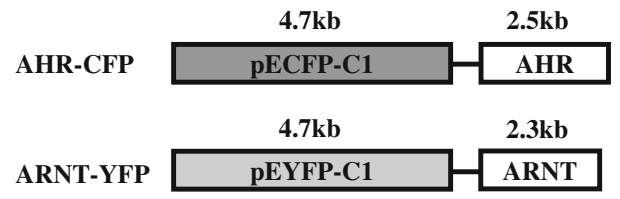

C Agarose gel electrophoresi s analysis of constructs

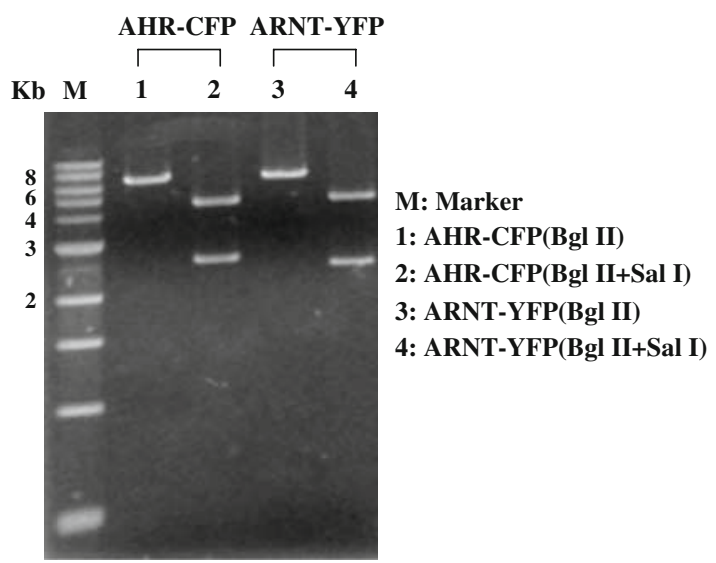

D Images of Constructs-tra nsfected H4IIEC3 cells

AHR-CFP
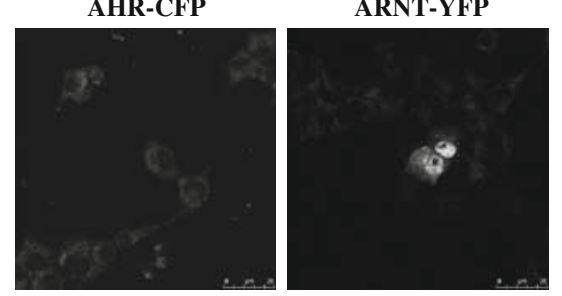

(c) Agarose gel electrophoresis analysis of the AHR-CFP plasmid construct digested with Bgl II (lane 1) or both Bgl II and Sal I (lane 2). Lanes 3 and 4 represent the ARNT-CFP plasmid construct digested by $\mathrm{Bgl}$ II or both $\mathrm{Bgl}$ II and Sal I, respectively. (d) Constructed AHR-CFP and ARNT-YFP plasmids were transfected into H4IIEC 3 cells. After $24 \mathrm{~h}$, cells were fixed with $4 \%$ paraformaldehyde solution, and then subjected to confocal microscopy by using CFP and YFP channel, respectively

after transfection and maintained in DMEM supplemented with $400 \mu \mathrm{l} / \mathrm{ml} \mathrm{G} 418$ (neomycin, Sigma, St. Louis, MO). Clonal expression was examined initially by fluorescence microscopy, and clones for further study were selected and expanded.

\section{Chemical preparation}

2,3,7,8-Tetrachlorodibenzo-p-dioxin was purchased from Boehringer Mannheim (Mannheim, Germany). The DMSO solution was obtained from Sigma (St. Louis, MO). The 2,3,7,8-tetrachlorodibenzo-p-dioxin solution was dissolved in the DMSO solution and diluted to different concentrations as indicated.

\section{Confocal microscopy}

Confocal microscopic cell images from transfected cells were obtained using a Leica TCS SP5 Confocal Spectral Microscope Imaging System (Leica, Heidelberg, Germany) expressing AHR-CFP, cells were diluted and seeded 2 days 
equipped with a 100-mW argon blue laser. All images were digitally processed using FRET AF software (Leica).

\section{FRET measurements}

FRET experiments were performed on the Leica TCS SP5 Confocal Spectral Microscope Imaging System. In protoplasts, the FRET signal was detected in a range between 520 and $580 \mathrm{~nm}$ with 458-nm excitation (FRET channel). CFP-crosstalk was determined by detecting the emission intensity in the range of 462-510 nm (CFP channel). Direct excitation of YFP was quantified by reference measurements of YFP fluorescence comparing the emission intensities at the excitation wavelengths of 458 and $514 \mathrm{~nm}$, respectively. As an additional control, the YFP fluorescence in the range of $520-580 \mathrm{~nm}$ was detected following excitation at $514 \mathrm{~nm}$ (YFP channel). For FRET measurements, an oil immersion objective with 40-fold magnification and a numerical aperture (NA) of 1.4 were used. The scan speed was $400 \mathrm{~Hz}$, and the image resolution was $512 \times 512$ pixels. For each set of transfections, each experiment was independently repeated.

\section{FRET quantification}

FRET signals were measured by acceptor photobleaching method according to a previous study [29]. The prebleach mCerulean and mCitrine images of transfected cells were sequentially collected through CFP channel (3\% intensity) and YFP channel ( $0.2 \%$ intensity) as baseline, respectively. The cells were then bleached by scanning a selected region of interest 20 times for 5-10 s using the $515 \mathrm{~nm}$ argon laser line at $20 \%$ intensity. Post-bleach mCerulean and mCitrine images were collected immediately following photobleaching using the same parameters as those used for acquiring prebleach images.

For FRET quantification, a method for detecting FRET signals through the sensitized acceptor emission was followed [29], and the FRET efficiency, $\mathrm{E}_{\mathrm{A}}(\mathrm{i})$ was calculated as a percentage increase in mCerulean fluorescence intensity following the mCitrine photobleaching using the formula:

$\mathrm{E}_{\mathrm{A}}(\mathrm{i})=\left[\mathrm{F}^{\mathrm{DA}}(\mathrm{i})-\mathrm{F}^{\mathrm{D}}(\mathrm{i}) * \mathrm{R}_{\mathrm{D}}-\mathrm{F}^{\mathrm{A}}(\mathrm{i}) * \mathrm{R}_{\mathrm{E}}\right] / \mathrm{F}^{\mathrm{A}}(\mathrm{i}) ;$

where $F^{D A}(i)$ is obtained by the sensitized emission, $F^{D}(i)$ is a measure of the fluorescence through the donor filter upon donor excitation, $\mathrm{F}^{\mathrm{A}}(\mathrm{i})$ is a measure of the acceptor emission upon acceptor excitation, $R_{D}$ is the ratio of the detection efficiencies of the donor emission intensities through the acceptor and donor filter sets, and $R_{E}$ is the ratio of the extinction coefficients of the acceptor when excited at the acceptor and donor wavelengths. Algebraic i is defined as each pixel of an image. The mean average and standard deviation of the FRET efficiency of each set of measurements were calculated.

\section{Results}

Characterization of CFP- and YFP-tagged AHR and ARNT constructs

To evaluate the fluorescence energy transfer between mCerulean and mCitrine, we first established CFP- and YFPtagged AHR and ARNT constructs, the AHR-CFP and ARNT-YFP plasmids (Fig. 1b). As shown in Fig. 1c, the AHR-CFP plasmid treated with Bgl II generated a single 7.2$\mathrm{kb}$ band, which corresponded to the size of the PECFP-C1 vector encoding the full-length human AHR gene. Moreover, AHR-CFP co-digested by Bgl II and Sal I generated two bands of 4.7 and $2.5 \mathrm{~kb}$, which corresponded to the size of the PECFP-C1 vector, respectively. Similar results were observed with ARNT-YFP plasmids digested by these restriction enzymes as indicated (Fig. 1c). Furthermore, CFP or YFP signals were observed in the cell cytoplasm and nuclei in AHR-CFP- or ARNT-YFP-transfected H4IIEC3 cells, indicating that these two constructed plasmids were successfully expressed in H4IIEC3 cells (Fig. 1d). These results confirmed that the AHR-CFP and ARNT-YFP plasmids were well-constructed and functioned properly; thus, they are available as FRET probes in H4IIEC3 cells.

\section{Exposure to TCDD induces FRET signals} in H4IIEC3 cells

H4IIEC3 cells were co-transfected with the AHR-CFP and ARNT-YFP plasmids. Twenty-four hours after transfection, H4IIEC 3 cells were treated with 0 or $10 \mathrm{pM}$ TCDD. As shown in Fig. 2a, no FRET signal was detected with 0-pM TCDD treatment. However, the FRET signal significantly increased with 10-pM TCDD treatment. Moreover, the TCDD-upregulated FRET signal was observed in cell nuclei (Fig. 2, bottom panel). The signal intensity of CFP or YFP, which was used to monitor the transfection efficiency did not significantly differ in either the 0 - or 10-pM TCDD sample (Fig. 2, upper and middle panels). The intensities of the FRET signals were quantified and are shown in Fig. $2 b$. Quantification results indicated that treatment with $10 \mathrm{pM}$ TCDD produced 1.8-fold FRET signal induction compared to 0-pM TCDD treatment in H4IIEC3 cells (Fig. 2b). As shown in Fig. 2c, the FRET signal was also significantly enhanced by 10 pM TCDD treatment in H4IIEC3 cells stably expressed AHR-CFP followed by transient transfected with ARNT-YFP construct (Fig. 2c). The treatment with $10 \mathrm{pM}$ TCDD also exhibited 2.6-fold FRET signal induction compared to $0 \mathrm{pM}$ TCDD treatment in these double 
Fig. 2 TCDD enhanced FRET signal elevation in AHR-CFPand ARNT-YFP- transfected H4IIEC3 cells. (a) AHR-CFP and ARNT-YFP constructs were transfected into H4IIEC 3 cells. After $24 \mathrm{~h}$, cells were treated with 0 (left panel) or $10 \mathrm{pM}$ TCDD (right panel) for $1 \mathrm{~h}$. Cells were fixed with $4 \%$ paraformaldehyde solution for $30 \mathrm{~min}$ and then subjected to confocal microscopy using the following channels: donor-CFP (upper panel), acceptor-YFP (middle panel), and FRET (bottom panel). (b) The histogram presents quantification of the FRET signals. (c) ARNT-YFP constructs was transfected into H4IIEC3 cells stably expressing AHR-CFP. Cells were treated with 0 or $10 \mathrm{pM}$ TCDD for $1 \mathrm{~h}$. Cells were fixed with $4 \%$ paraformaldehyde solution for $30 \mathrm{~min}$ and then subjected to confocal microscopy using channels: of donor-CFP, acceptor-YFP, and FRET. (d)

The histogram presents quantification of the FRET signals. The data are representative of three experiments and are expressed as the mean $\pm \mathrm{SE}$
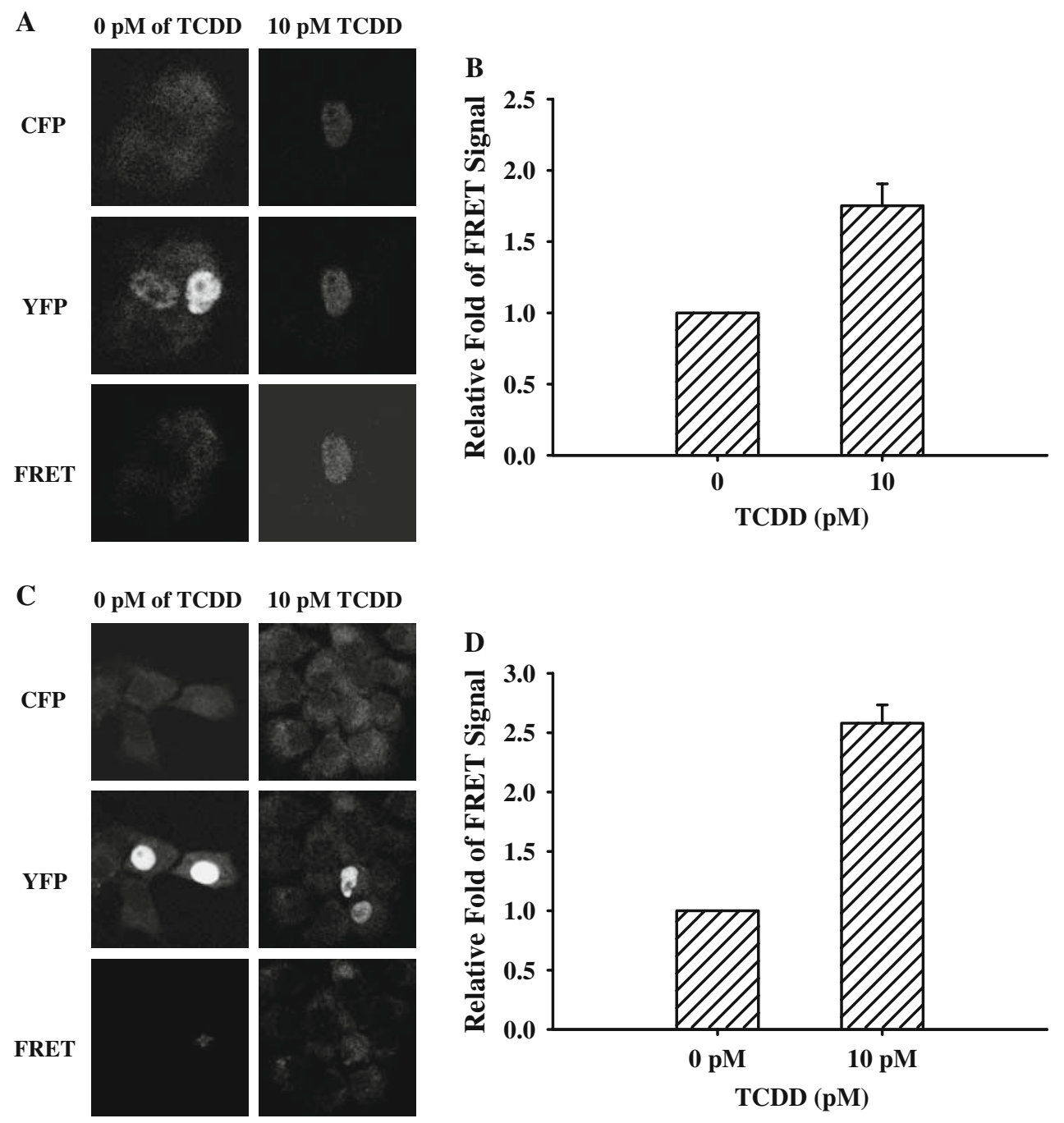

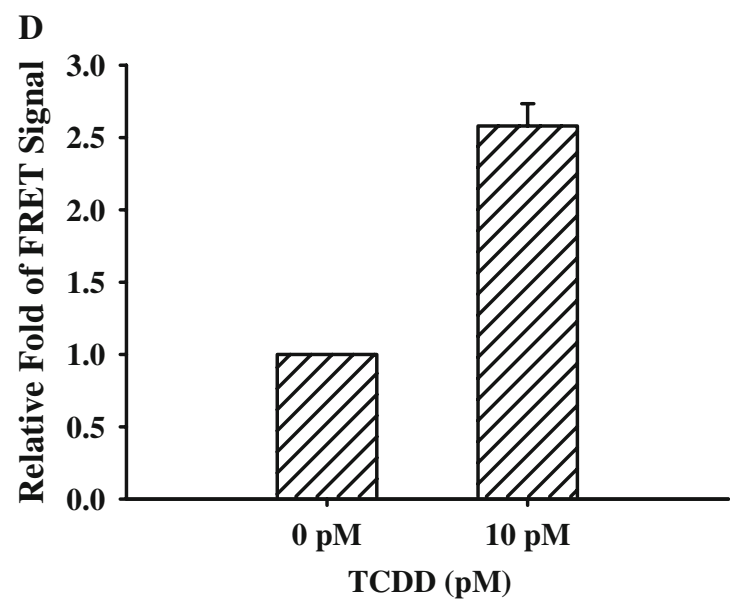

transfected cells (Fig. 2d). These results indicated that the data obtained in transient transfected cell lines exert similar results as those obtained in stable cell lines.

Exposure to dioxin induces FRET signals in H4IIEC3 cells in a dose-dependent manner

Since TCDD showed significant enhancement effects on FRET signals in H4IIEC3 cells, we further investigated if TCDD suppressed FRET signals in H4IIEC 3 cells in a dosedependent manner. H4IIEC3 cells co-transfected with the AHR-CFP and ARNT-YFP plasmids were treated with 0,1 , 10, 50, 100, or 1,000 pM TCDD. The FRET signal was not observed with 0-pM TCDD treatment. Subsequently, FRET signals were first significantly enhanced with 1 pM TCDD, and they increased thereafter and peaked at 1,000 pM (Fig. 3a). The intensity of FRET signals were quantified and are shown in Fig. 3b. These results indicated that treatment with TCDD enhanced FRET signal elevation in a dosedependent manner in H4IIEC3 cells (Fig. 3b).

\section{Discussion}

Our results clearly showed that transient expression of AHR and ARNT respectively encoding CFP and YFP proteins could result in FRET signals in response to dioxin treatment in H4IIEC3 cells (Fig. 2). These results are consistent with previous results that AHR and ARNT can form a heterodimer in response to TCDD [30]. With treatment with $0 \mathrm{pM}$ TCDD, the CFP signals representative of AHR were detected in the cell cytoplasm of H4IIEC3 cells. However, this signal was subsequently detected in the nucleus after 10 pM TCDD treatment (Fig. 2). These results indicated that treatment with dioxin induced AHR translocation from the cell cytoplasm to the nucleus, which is consistent with a previous study [31]. Moreover, YFP signals representative of ARNT were detected in the cell nucleus in the absence and presence of dioxin (Fig. 2). These results are consistent with a previous study that ARNT is dominantly expressed in the cell nucleus, which enables it to interact with AHR to form a heterodimer 

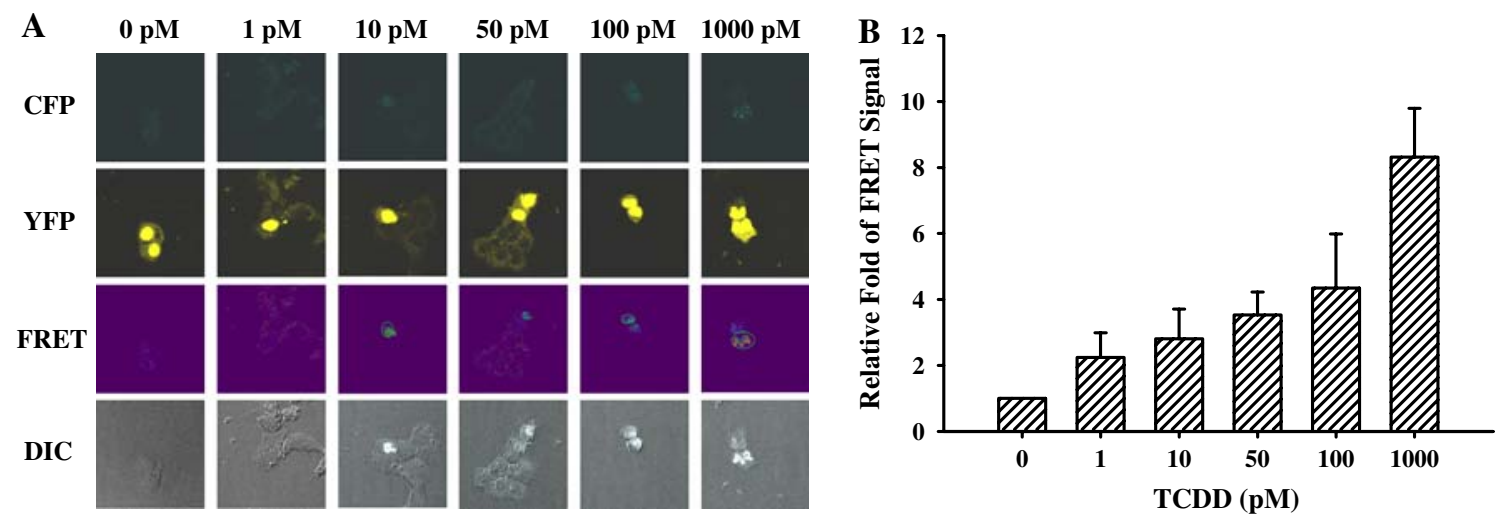

Fig. 3 TCDD enhanced FRET signal elevation in AHR-CFP- and ARNT-YFP-transfected H4IIEC3 cells in a dose-dependent manner. (a) AHR-CFP and ARNT-YFP constructs were transfected into H4IIEC3 cells. After $24 \mathrm{~h}$, cells were fixed, treated with $0,10,50$, 100 , or $1,000 \mathrm{pM}$ of TCDD for $1 \mathrm{~h}$, and then subjected to confocal

complex in the presence of dioxin [32]. In addition, TCDDinduced FRET signals were detected in the cell nucleus in the presence of TCDD, further confirming that an AHRARNT complex forms in the cell nucleus (Fig. 2).

A previous study indicated that the liver is one of the most sensitive organs compared to other organs in the body [33]. A mouse hepatoma cell-based CYP1A1-CALUX bioassay was successfully established [23]. The hepatoma cell line can be representative of liver cells, and its immortalized characteristic is suitable for use as material for establishing a bioassay system. H4IIEC3 cells are rat hepatoma cells that were used for dioxin biological detection in our previous study [22]. In the present study, we expressed mouse AHR-CFP and ARNT-YFP fusion proteins in H4IIEC 3 cells to successfully avoid cross-specific interactions, and provide sensitization to FRET signal detection.

A previous study indicated that CFP and YFP are suitable for FRET detection [34]. To avoid CFP or YFP signal interference, FRET signals were normalized to CFP- or YFP-induced FRET signals. Moreover, our results indicated that dioxin treatment showed no effects on individual CFP or YFP signals. These data confirmed that dioxin does not alter the expression levels of AHR or ARNT, consistent with a previous study [15].

In the present study, we showed that TCDD induced FRET signals in a dose-dependent manner. TCDD-induced FRET signal induction was first observed at $1 \mathrm{~h}$, indicating that this FRET-based dioxin detection system is a rapid detection system compared to previously established dioxin detection systems. In addition, TCDD-induced FRET signal induction was first observed with 1-pM treatment, indicating that the sensitivity of this detection system might reach the 1pM level. These results further imply that this FRET-based dioxin detection system is quite sensitive compared to other microscopy using the following channels: donor-CFP (upper panel), acceptor-YFP (middle panel), and FRET (bottom panel). (b) The histogram presents quantification of the FRET signals. Data are representative of three experiments and are expressed as the mean $\pm \mathrm{SE}$

detection systems (Fig. 3). Our established FRET-based dioxin bioassay is time-effective and highly sensitive, and represents a great improvement over other dioxin detection systems reported previously.

By the DRE-CALUX bioassay, dose-response results of 2,3,7,8-TCDD represents good correlation of up to $r^{2}=0.890$ [23]. Similar results were also obtained from other studies $[25,35,36]$. Our results demonstrated that the FRET-based bioassay for 2,3,7,8-TCDD detection also showed a good correlation of up to $r^{2}=0.863$ (Fig. 3), indicating that this technique also has as high an accuracy as other bioassays reported before. The sensitivity of the DRE-CALUX bioassay as well as FRET-based detection system can reach the 1-pM level [23]. However, to obtain results from the DRE-CALUX bioassay takes at least $24 \mathrm{~h}$. Since the AHR and ARNT interaction is situated upstream of CYP1A1 promoter activation, the operation time of FRET-based detection system evaluated through AHR and ARNT association requires only $1 \mathrm{~h}$. Our data suggested that the FRET-based detection system might be the most time-efficient bioassay.

Accumulating evidence indicates that not only TCDD derivatives but also PCBs and other PAH toxicants affect the AHR-mediated signaling transduction pathway [3]. Therefore, identification of toxicant types cannot rely on bioassays other than the HRGC/MS method. Joung et al. reported that DRE-CALUX can serve as a high-throughput pre-screening method for dioxin-like compounds in environmental matrices. Selecting suspect samples subjected to the HRGC/MS method can accurately confirm its concentration and chemical characteristics [23]. Our results showed that the FRETbased bioassay might be an alternative high-throughput dioxin pre-screening method for dioxin detection which is more time-effective than the DRE-CALUX bioassay. In this study, we have demonstrated that FRET-based dioxin 
Principle of FRET-based Bioassay for detecting dioxin-like compounds

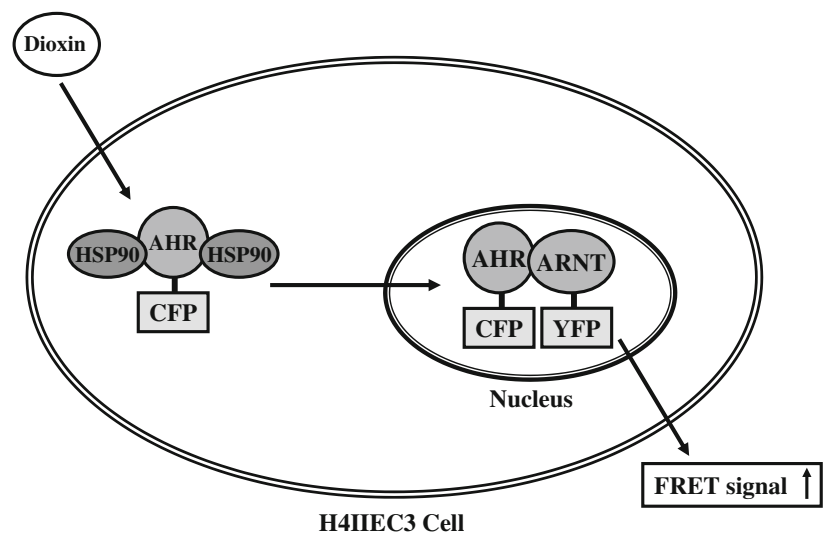

Fig. 4 Determination of TCDD concentration through detecting FRET signals in AHR-CFP- and ARNT-YFP-co-expressing H4IIEC3 cells

detection system may serve as a rapid preliminary screening method for dioxin-like compound.

Intensity based FRET measurements carried out by confocal fluorescence microscopy analysis has been reported in recent study [37]. Our present results also suggested that the FRET measurements carried out by confocal fluorescence microscopy analysis is feasible. Recent report confirmed that quantification of FRET signal carried out by two-photon fluorescence lifetime imaging microscopy (TPFLIM) can be quantitatively measured with high sensitivity [38]. Moreover, the quantifications of FRET signals by a conventional fluorescence microscope plus cooled CCD camera might allow signal integration to achieve improved contrast. These methods might be used as alternatives to enhance FRET signals in our system in the future.

Our results demonstrated the suitability of the FRETbased dioxin detection system. Co-expression of AHR-CFP and ARNT-YFP in H4IIEC3 cells successfully exhibited FRET signals in response to dioxin treatment (Fig. 4). Moreover, these enhancement effects occurred in a dosedependent manner, which had a good correlation with high $r^{2}$ values. Compared to the CALUX-or chemistry-based dioxin detection systems, the FRET-based dioxin detection system showed greater time efficiency, economy, and sensitivity. For future practical utility of FRET-based dioxin detection systems, co-expression of an AHR-CFP and ARNT-YFP stable cell line will be generated, which can contribute to large-scale screening of suspected dioxincontaminated samples. Our established FRET-based method for detecting dioxin profoundly improved on disadvantages of other detection systems. The use of FRET as a dioxin-detection bioassay was successful and is suitable for an initial screening for dioxin-like compounds.
Acknowledgements This work was supported by grants NSC962311-B-002-018-MY2 and NSC95-2752-B-006-003-PAE (to H. Lee) from the National Science Council, Taiwan. We thank the excellent technical assistance of Technology Commons, College of Life Science, National Taiwan University with confocal microscopy.

\section{References}

1. Safe S (1990) Polychlorinated biphenyls (PCBs), dibenzo-p-dioxins (PCDDs), dibenzofurans (PCDFs), and related compounds: environmental and mechanistic considerations which support the development of toxic equivalency factors (TEFs). Crit Rev Toxicol 21:51-88

2. Hu K, Bunce NJ (1999) Metabolism of polychlorinated dibenzop-dioxins and related dioxin-like compounds. J Toxicol Environ Health B Crit Rev 2:183-210

3. Van den Berg M, Birnbaum LS, Denison M, De Vito M, Farland W, Feeley M, Fiedler H, Hakansson H, Hanberg A, Haws L, Rose M, Safe S, Schrenk D, Tohyama C, Tritscher A, Tuomisto J, Tysklind M, Walker N, Peterson RE (2006) The 2005 World Health Organization reevaluation of human and mammalian toxic equivalency factors for dioxins and dioxin-like compounds. Toxicol Sci 93:223-241

4. Hay A (1978) Dioxin source is safe. Nature 274:526

5. Parzefall W (2002) Risk assessment of dioxin contamination in human food. Food Chem Toxicol 40:1185-1189

6. Gregoraszczuk EL (2002) Dioxin exposure and porcine reproductive hormonal activity. Cad Saude Publica 18:453-462

7. Cook JC, Gaido KW, Greenlee WF (1987) Ah receptor: relevance of mechanistic studies to human risk assessment. Environ Health Perspect 76:71-77

8. Eriksson M, Hardell L, Adami HO (1990) Exposure to dioxins as a risk factor for soft tissue sarcoma: a population-based casecontrol study. J Natl Cancer Inst 82:486-490

9. Fingerhut MA, Halperin WE, Honchar PA, Smith AB, Groth DH, Russell WO (1984) An evaluation of reports of dioxin exposure and soft tissue sarcoma pathology among chemical workers in the United States. Scand J Work Environ Health 10:299-303

10. McGregor DB, Partensky C, Wilbourn J, Rice JM (1998) An IARC evaluation of polychlorinated dibenzo-p-dioxins and polychlorinated dibenzofurans as risk factors in human carcinogenesis. Environ Health Perspect 106:755-760

11. Tondeur Y, Niederhut WN, Campana JE, Missler SR (1987) A hybrid HRGC/MS/MS method for the characterization of tetrachlorinated-p-dioxins in environmental samples. Biomed Environ Mass Spectrom 14:449-456

12. Fernandezsalguero P, Pineau T, Hilbert DM, McPhail T, Lee SST, Kimura S, Nebert DW, Rudikoff S, Ward JM, Gonzalez FJ (1995) Immune system impairment and hepatic fibrosis in mice lacking the dioxin-binding Ah receptor. Science 268:722-726

13. Schmidt JV, Su GHT, Reddy JK, Simon MC, Bradfield CA (1996) Characterization of a murine Ahr null allele: involvement of the Ah receptor in hepatic growth and development. Proc Natl Acad Sci USA 93:6731-6736

14. Backlund M, Johansson I, Mkrtchian S, Ingelmansundberg M (1997) Signal transduction-mediated activation of the aryl hydrocarbon receptor in rat hepatoma H4IIE cells. J Biol Chem 272:31755-31763

15. Roberts BJ, Whitelaw ML (1999) Degradation of the basic helixloop-helix/Per-ARNT-Sim homology domain dioxin receptor via the ubiquitin/proteasome pathway. J Biol Chem 274:3635136356

16. Giannone JV, Li W, Probst M, Okey AB (1998) Prolonged depletion of $\mathrm{AH}$ receptor without alteration of receptor mRNA levels 
after treatment of cells in culture with 2,3,7,8-tetrachlorodibenzop-dioxin. Biochem Pharmacol 55:489-497

17. Ma Q, Baldwin KT (2000) 2,3,7,8-Tetrachlorodibenzo-p-dioxininduced degradation of aryl hydrocarbon receptor (AhR) by the ubiquitin-proteasome pathway-role of the transcription activaton and DNA binding of AhR. J Biol Chem 275:8432-8438

18. Tsai JC, Perdew GH (1997) Ah receptor nuclear translocator protein heterogeneity is altered after heterodimerization with the Ah receptor. Biochemistry 36:9066-9072

19. Stanker LH, Watkins B, Rogers N, Vanderlaan M (1987) Monoclonal antibodies for dioxin: antibody characterization and assay development. Toxicology 45:229-243

20. Okuyama M, Kobayashi N, Takeda W, Anjo T, Matsuki Y, Goto J, Kambegawa A, Hod S (2004) Enzyme-linked immunosorbent assay for monitoring toxic dioxin congeners in milk based on a newly generated monoclonal anti-dioxin antibody. Anal Chem 76:1948-1956

21. Postlind H, Vu TP, Tukey RH, Quattrochi LC (1993) Response of human CYP1-luciferase plasmids to 2,3,7,8-tetrachlorodibenzop-dioxin and polycyclic aromatic hydrocarbons. Toxicol Appl Pharmacol 118:255-262

22. Huang YW, Lee WS, Chang-Chien GP, Lin CI, Kao CY, Lee H (2004) Establishment of cellular detection systems for dioxin-like compounds. Chin Biosci 47:1-18

23. Joung KE, Chung YH, Sheen YY (2007) DRE-CALUX bioassay in comparison with HRGC/MS for measurement of toxic equivalence in environmental samples. Sci Total Environ 372:657-667

24. Bradlaw JA, Garthoff LH, Hurley NE, Firestone D (1980) Comparative induction of aryl hydrocarbon hydroxylase activity in vitro by analogs of dibenzo-p-dioxin. Food Cosmet Toxicol 18:627-635

25. Sanderson JT, Aarts J, Brouwer A, Froese KL, Denison MS, Giesy JP (1996) Comparison of Ah receptor-mediated luciferase and ethoxyresorufin-O-deethylase induction in H4IIE cells: implications for their use as bioanalytical tools for the detection of polyhalogenated aromatic hydrocarbons. Toxicol Appl Pharmacol 137:316-325

26. Griesbeck O, Baird GS, Campbell RE, Zacharias DA, Tsien RY (2001) Reducing the environmental sensitivity of yellow fluorescent protein-mechanism and applications. J Biol Chem 276:29188-29194

27. Seidel T, Golldack D, Dietz KJ (2005) Mapping of C-termini of $\mathrm{V}$-ATPase subunits by in vivo-FRET measurements. FEBS Lett 579:4374-4382
28. Khan S, Barhoumi R, Burghardt R, Liu SX, Kim K, Safe S (2006) Molecular mechanism of inhibitory aryl hydrocarbon receptorestrogen receptor/Sp1 cross talk in breast cancer cells. Mol Endocrinol 20:2199-2214

29. Karpova TS, Baumann CT, He L (2003) Fluorescence resonance energy transfer from cyan to yellow fluorescent protein detected by acceptor photobleaching using confocal microscopy and a single laser. J Microsc 209:56-70

30. Kobayashi A, Sogawa K, Fujiikuriyama Y (1996) Cooperative interaction between AhR center dot Arnt and Sp1 for the druginducible expression of CYP1A1 gene. J Biol Chem 271:12310 12316

31. Probst MR, Reiszporszasz S, Agbunag RV, Ong MS, Hankinson O (1993) Role of the aryl hydrocarbon receptor nuclear translocator protein in aryl hydrocarbon (dioxin) receptor action. Mol Pharmacol 44:511-518

32. Hankinson O (1995) The aryl hydrocarbon receptor complex. Annu Rev Pharmacol Toxicol 35:307-340

33. Barbieri S, Pirovano C, Scarlato G, Tarchini P, Zappa A, Maranzana M (1988) Long-term effects of 2,3,7,8-tetrachlorodibenzo-p-dioxin on the peripheral nervous system. Clinical and neurophysiological controlled study on subjects with chloracne from the Seveso area. Neuroepidemiology 7:29-37

34. Majoul I, Straub M, Duden R, Hell SW, Soling HD (2002) Fluorescence resonance energy transfer analysis of protein-protein interactions in single living cells by multifocal multiphoton microscopy. J Biotechnol 82:267-277

35. Murk AJ, Legler J, Denison MS, Giesy JP, vande Guchte C, Brouwer A (1996) Chemical-activated luciferase gene expression (CALUX): a novel in vitro bioassay for Ah receptor active compounds in sediments and pore water. Fundam Appl Toxicol 33:149-160

36. Zhang ZR, Xu SQ, Zhou YK, Xu YJ, Liu ZW, Cai XK, Tan XL (2002) Improvement of chemically-activated luciferase gene expression bioassay for detection of dioxin-like chemicals. Biomed Environ Sci 15:58-66

37. Lu J, Zhang Z, Yang J, Chu J, Li P, Zeng S, Luo Q (2007) Visualization of beta-secretase cleavage in living cells using a genetically encoded surface-displayed FRET probe. Biochem Biophys Res Commun 362:25-30

38. Murakoshi H, Lee SJ, Yasuda R (2008) Highly sensitive and quantitative FRET-FLIM imaging in single dendritic spines using improved non-radiative YFP. Brain Cell Biol 2008 May 30 (Epub ahead of print) 